\title{
Channel blockade in a two-path triple-quantum-dot system
}

\author{
M. Kotzian,,$^{1, *}$ F. Gallego-Marcos, ${ }^{2,1}$ G. Platero, ${ }^{2}$ and R. J. Haug $^{1}$ \\ ${ }^{1}$ Institut für Festkörperphysik, Leibniz Universität Hannover, Appelstrasse 2, 30167 Hannover, Germany \\ ${ }^{2}$ Instituto de Ciencia de Materiales, CSIC, Cantoblanco, 28049 Madrid, Spain \\ (Received 14 July 2015; revised manuscript received 10 June 2016; published 26 July 2016)
}

\begin{abstract}
Electronic transport through a two-path triple-quantum-dot system with two source leads and one drain is studied and the interaction between the two paths is analyzed. We observe a channel blockade as a result of interchannel Coulombic interaction. The experimental results are understood with the help of a theoretical model which allows one to obtain the parameters of the system, the stability regions of each state, and the full dynamical transport in the triple-dot resonances.
\end{abstract}

DOI: 10.1103/PhysRevB.94.035442

Triple quantum dots (TQDs), which have been implemented only recently [1-4], offer the possibility of analyzing new fascinating properties which are not present in doublequantum-dot systems. These new properties, to name a few, include interference phenomena between different transport channels giving rise to dark states in triangular [5-8] and linear [9] dot distributions and long distant coherent states in TQDs [9-13]. TQDs are, as the smallest qubit chain, a step towards the more complex architectures needed in quantum computation. They allow for novel applications in the field of quantum information processing, for example, as exchange-controlled spin qubits $[14,15]$ or as current rectifiers $[1,16]$. They provide as well the implementation of quantum cellular automata processes, a combination of charging and reconfiguration events in the system being a crucial process in quantum information [17,18]. Coherent electron transfer using adiabatic passage was proposed for TQDs in series [19]. Furthermore, decoherence due to charge fluctuations is reduced in a TQD-based coded qubit as it involves a decoherence-free subspace [15,20].

Our system is a triangular-shaped TQD with one lead attached to each dot and two of the dots only capacitively coupled [Fig. 1(a)]. A triangular geometry is suitable for studying entanglement and effects of interference which makes it an interesting device for quantum information processing. The flexibility of this setup makes it a convenient tool for investigating the transport properties of a TQD. In particular we study the interaction between two double dot paths within the TQD structure. Transport can be measured separately and simultaneously for the two double dot paths and be compared or combined to study the whole TQDs physics on the basis of the double dots.

In contrast to former published works [4] where one source and two drain leads were used, we now use one drain and two source leads. In this configuration of two-path transport the electrons from the different paths compete for the occupation of $\operatorname{dot} \mathrm{A}$, which is shared by both paths [Fig. 1(a)]. This competition gives rise to a current-blocking effect, produced by the interchannel Coulombic interaction. By applying different ac frequencies with a lock-in to the paths, the interaction between the paths becomes apparent in a more distinct manner.

*kotzian@nano.uni-hannover.de
We analyze the role of interactions between the charge flowing through the two different paths by transport measurements. We observe, as a consequence of interchannel Coulombic interaction, channel blockade in transport.

\section{TQD SAMPLE AND CHARACTERIZATION}

The measurements were performed on a lateral TQD made with local anodic oxidation by atomic force microscopy (AFM) on a GaAs/AlGaAs heterostructure [21-23]. A twodimensional electron gas with an electron concentration of $n_{e}=3.47 \times 10^{15} \mathrm{~m}^{-2}$ is located at $33 \mathrm{~nm}$ depth below the surface. The dots A,B,C are arranged in a triangular geometry [4] with each dot placed next to the other two and one lead attached to each dot [Fig. 1(a)]. Dots A and B and also A and $\mathrm{C}$ are tunnel coupled; dots $\mathrm{B}$ and $\mathrm{C}$ are only capacitively coupled. The source leads $S_{1}$ and $S_{2}$ are connected to dots $\mathrm{B}$ and $\mathrm{C}$, respectively, and dot $\mathrm{A}$ is connected to the drain lead D. We have two transport paths: path 1 with dots A and $\mathrm{B}$ and path 2 with dots $\mathrm{A}$ and $\mathrm{C}$. The sample has four in-plane gates $G_{1}-G_{4}$ [Fig. 1(b)] to control the potential of the dots, interdot, and dot-lead couplings. A quantum point contact (QPC) sensitive to all three dots is placed next to dots $\mathrm{B}$ and $\mathrm{C}$ to perform charge measurements. The measurements were conducted in a dilution refrigerator. To measure the differential conductance of the two transport paths simultaneously but separately, a lock-in technique was used with ac voltages with two different frequencies $f_{1}=83.3 \mathrm{~Hz}$ and $f_{2}=18.3 \mathrm{~Hz}$, with $U_{\mathrm{AC}}=10 \mu \mathrm{V}$ applied to $\mathrm{S}_{1}$ and $\mathrm{S}_{2}$, respectively. In addition, different dc voltages are applied to the source contacts. The QPC was operated by applying a dc voltage to the source of the $\mathrm{QPC}, S_{\mathrm{QPC}}$, and measuring a dc current at the drain of the QPC, $D_{\mathrm{QPC}}$. The QPC is tuned by the gate $G_{\mathrm{QPC}}$. In our transport measurement range the dots contain several tens of electrons on the whole. The charging energies are $E_{\mathrm{ch}, \mathrm{A}}=2 \mathrm{meV}, E_{\mathrm{ch}, \mathrm{B}}=6 \mathrm{meV}$, and $E_{\mathrm{ch}, \mathrm{C}}=3$ meV for dots $\mathrm{A}, \mathrm{B}$, and $\mathrm{C}$, respectively.

\section{A. Charge measurements}

To characterize the device, the charging is studied by using the QPC as a detector. The derivative of the QPC current is plotted as a function of gate voltages $U_{\mathrm{G} 1}$ and $U_{\mathrm{G} 3}$ (Fig. 2) with denoted charge configurations $\left|N_{\mathrm{A}}, N_{\mathrm{B}}, N_{\mathrm{C}}\right\rangle$, where $N_{\mathrm{i}}$ are the occupations of dots A,B,C. The electrons in the core of the 
(a)

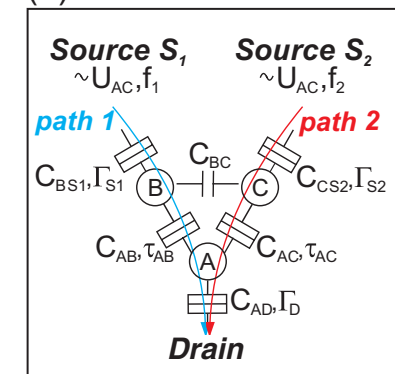

(b)
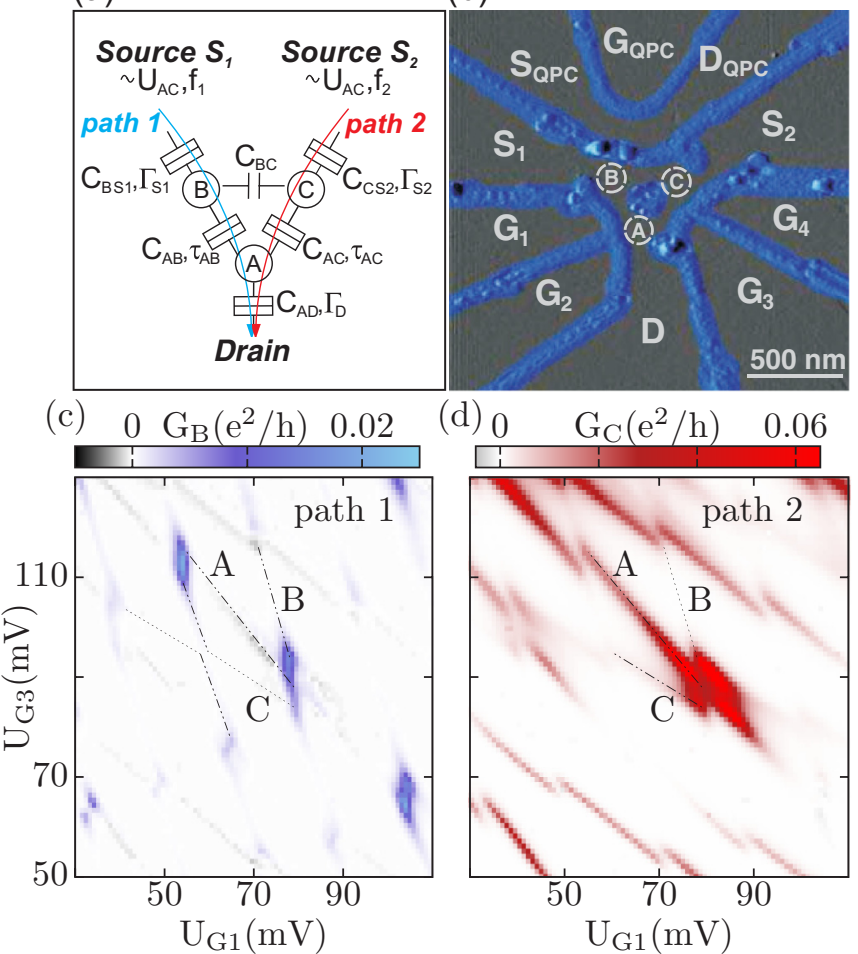

(d) 0

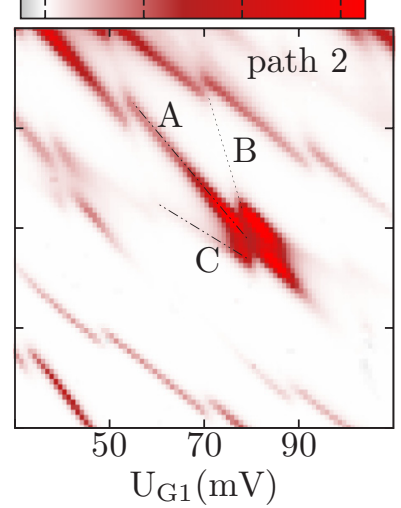

FIG. 1. (a) Schematic of the TQD setup with capacitive and tunnel couplings. (b) AFM picture of the TQD sample with the in-plane gates $\mathrm{G}_{1}-\mathrm{G}_{4}$ and a QPC for charge measurements. The blue lines indicate the insulating barriers written by AFM. (c) Transport through path 1. Charging lines of dots A and B (dot-dashed lines) are observed. Charging of $\operatorname{dot} \mathrm{C}$ (dotted line) is observed by a shift of the charging lines. (d) Transport through path 2. Charging lines of dot A and C (dot-dashed lines) can be seen and charging of dot B (dotted line) is observed by a shift of these charging lines.

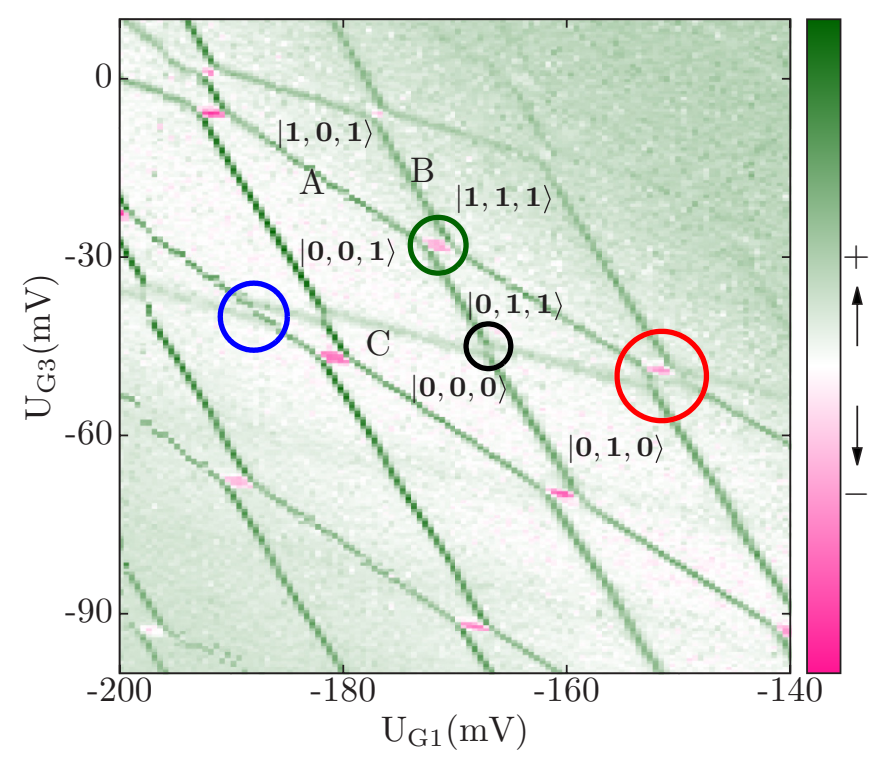

FIG. 2. Charge measurement using the QPC. The green lines with different slopes point out the charging of the respective dot with one electron. Double-dot resonances are marked by circles in green, black, and blue, the triple-dot resonance by a red circle. dots are not included in $N_{\mathrm{i}}$. The green lines indicate charging events, where one more electron is added to the system; pink lines indicate electron movement away from the detector. In Fig. 2 there are charging lines with three different slopes. Each line corresponds to one dot. The slope of the line depends on the capacitive coupling of the dot to the other dots and the gates $\mathrm{G}_{1}$ and $\mathrm{G}_{3}$. The lines with the lowest slope belong to $\operatorname{dot} \mathrm{C}$ as it is the least coupled to $U_{\mathrm{G} 1}$, the lines with intermediate slope to $\operatorname{dot} \mathrm{A}$ and the lines with the largest slope to $\operatorname{dot} \mathrm{B}$ as it is the least coupled to $U_{\mathrm{G} 3}$. Two dots are in resonance where two charging lines meet. In those resonances there are two degeneracy points among three different charge configurations, the so-called triple points (TPs). Charge reconfiguration lines connecting the two TPs mark the charge transitions between the dots.

Resonances between dot A and B (green circle), A and C (blue circle), and also between the only capacitively coupled dots $\mathrm{B}$ and $\mathrm{C}$ located in the two different paths (black circle) are observed [24]. In the red circle the three charging lines meet. This is a resonance among the three dots. We will focus on such a region in the transport measurements.

\section{B. Transport measurements}

To understand transport in this system, the differential conductance $G$ is measured along path 1 and 2 simultaneously, sweeping gate voltages $U_{\mathrm{G} 1}$ and $U_{\mathrm{G} 3}$ [Figs. 1(c) and 1(d)]. In doing so, the QPC is not in use. TPs with finite differential conductance are observed in both paths where the two dots are in resonance. In path 1 [Fig. 1(c)] resonances of the dots $\mathrm{A}$ and $\mathrm{B}$ can be seen. Charging of dot $\mathrm{C}$ is observed by a shift of the charging lines of dot $\mathrm{A}$ and $\mathrm{B}$, where $\operatorname{dot} \mathrm{C}$ comes into resonance. Analogously, charging lines of dot A and C appear in path 2 [Fig. 1(d)], and charging of dot B is detected indirectly by the shift of the charging lines of dots $\mathrm{A}$ and $\mathrm{C}$.

A triple-dot resonance is formed where two double-dot resonances coincide. In Fig. 3(a), where we combine path 1 and path 2 as observed in Figs. 1(c) and 1(d), we have three double-dot resonances, $\mathrm{A}$ and $\mathrm{B}, \mathrm{A}$ and $\mathrm{C}, \mathrm{B}$ and $\mathrm{C}$, in close vicinity to each other. One observes regions of high differential conductance in both paths but at different gate voltages $U_{\mathrm{G} 1}$ and $U_{\mathrm{G} 3}$. Along the B charging line (path 1 ) we can identify two resonance lines where $\mathrm{A}$ is resonant with $\mathrm{B}$. The whole triple-dot physics can be observed. The different occupations of the states at the TP participating in transport are marked in Fig. 3.

To understand the experimental results in more detail a theoretical model is developed to reproduce and explain the transport properties measured in the system. We fit the transport simulations to the experimental data to extract the interdot tunnel couplings $\tau_{\mathrm{AB}}=0.012 \mathrm{meV}, \tau_{\mathrm{AC}}=0.020 \mathrm{meV}$, the dot-lead tunnel couplings $\Gamma_{\mathrm{D}}=0.008 \mathrm{meV}, \Gamma_{\mathrm{S}_{1}}=0.003 \mathrm{meV}$, $\Gamma_{\mathrm{S}_{2}}=0.006 \mathrm{meV}$, and the electron temperature $T_{\mathrm{el}}=300 \mathrm{mK}$. In the model we distinguish between particles coming from $\mathrm{S}_{1}$ and $S_{2}$ in order to be as close as possible to the experimental conditions.

\section{THEORETICAL FRAMEWORK}

Here we discuss in detail the theory used to simulate the experiment. The total Hamiltonian of the system reads 

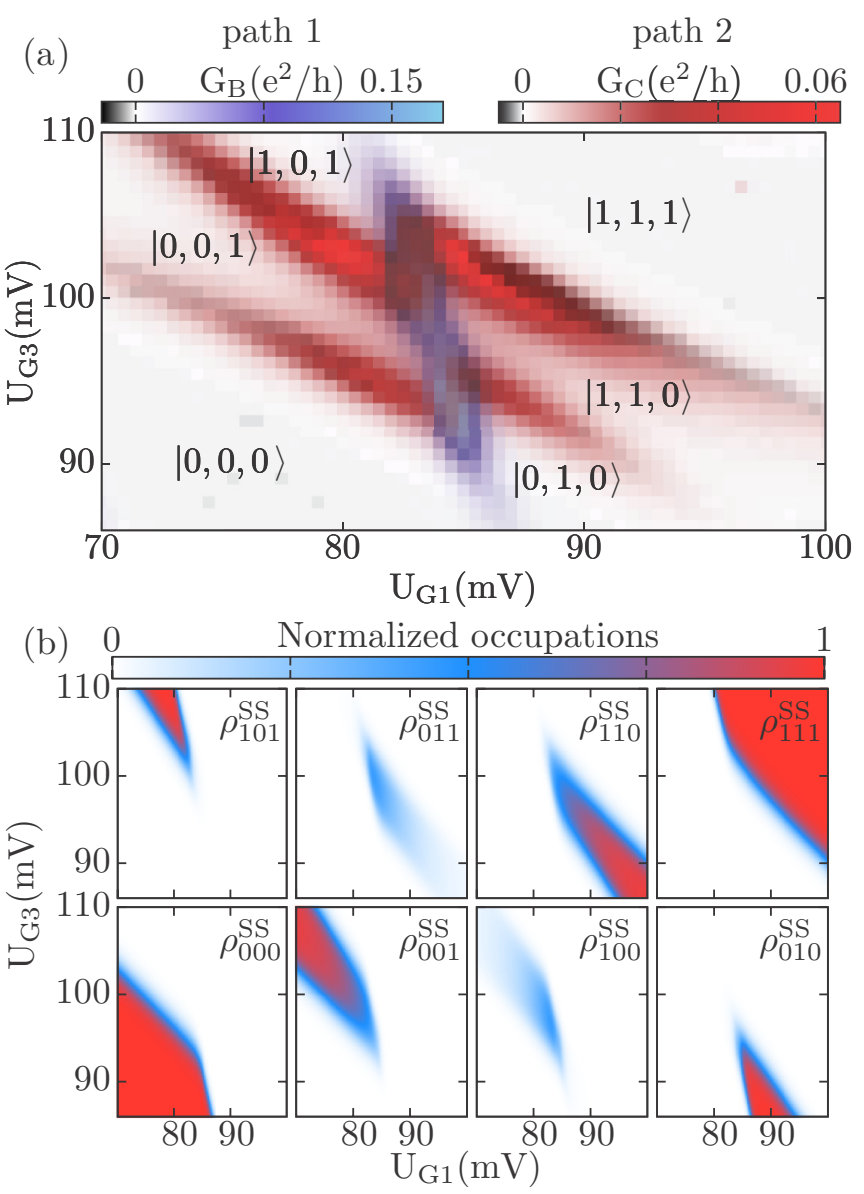

FIG. 3. (a) Experimental figure. Combined color plot of differential conductance through paths 1 and 2 with denoted charge configurations of the stability regions we were able to identify in the measurement. (b) Theoretical simulation. Occupation probabilities $\left(\rho_{i}^{\mathrm{SS}}\right)$ of the $i$ states present in the system. States $|1,0,0\rangle$ and $|0,1,1\rangle$ are within a small region with low occupation probability; their position in the stability diagram is not appreciable in the experimental figure (a).

$\hat{H}=\hat{H}_{0}+\hat{H}_{\text {lead }}+\hat{H}_{\text {int }}$. The dot system $\left(\hat{H}_{0}\right)$ is a threesite Anderson-like Hamiltonian [25]: $\hat{H}_{0}=\sum_{i} \epsilon_{i} \hat{c}_{i}^{\dagger} \hat{c}_{i}+$ $\sum_{i} \tau_{i, i+1} \hat{c}_{i}^{\dagger} \hat{c}_{i+1}+\sum_{i<j} V_{i j} \hat{n}_{i} \hat{n}_{j}$, where $\hat{c}_{i}^{\dagger}$ is the electron creation operator and $\hat{n}_{i}$ the particle number operator of dot $i . \epsilon_{i}(i \in\{\mathrm{A}, \mathrm{B}, \mathrm{C}\})$ is the chemical potential of the dots, $\tau_{i j}$ the coherent interdot tunnel coupling, and $V_{i j}$ the Coulomb interaction between the electrons in different dots. The reservoirs are modeled as a Fermi electron gas $\hat{H}_{\text {lead }}=$ $\sum_{l k} \varepsilon_{l k} \hat{d}_{l k}^{\dagger} \hat{d}_{l k}$ that has a constant temperature $T$ and chemical potential $\mu_{l}\left(l \in\left\{\mathrm{S}_{1}, \mathrm{~S}_{2}, D\right\}\right)$. The interaction part of the Hamiltonian $\hat{H}_{\text {int }}=\sum_{l i} \gamma_{l} \hat{d}_{l}^{\dagger} \hat{c}_{i}+$ H.c. couples the reservoirs and the dots with a hopping parameter $\gamma_{l}$. The energy levels are tuned with the gate voltages present in the experiment $\left(U_{\mathrm{G} 1}, U_{\mathrm{G} 2}, U_{\mathrm{G} 3}, U_{\mathrm{G} 4}\right)$.

The rates between the leads and the dots for incoming (+) and outgoing $(-)$ electrons with respect to the dot system are given by Fermi's golden rule $\Gamma_{i \leftarrow l}^{(+)}=2 \pi / \hbar\left|\gamma_{l}\right|^{2} f\left(\mu_{l}-\epsilon_{i}\right)$ and $\Gamma_{l \leftarrow i}^{(-)}=2 \pi / \hbar\left|\gamma_{l}\right|^{2}\left[1-f\left(\mu_{l}-\epsilon_{i}\right)\right]$, where $f$ is the Fermi distribution function. $\Gamma_{l} \equiv 2 \pi / \hbar\left|\gamma_{l}\right|^{2}$ is smaller than the interdot coupling $\tau_{i j}$; thus we can apply the Born-Markov approximation [26] for the interaction of the system with the leads. From the von Neumann equation $\partial_{t} \varrho(t)=i / \hbar[\hat{H}, \varrho(t)]$, which contains the full system time evolution, we trace over the bath's degrees of freedom, getting the reduced density matrix $\rho(t)=\operatorname{Tr}_{\text {leads }} \varrho(t)$ [27] and obtaining the master equation

$$
\partial_{t} \rho_{i}(t)=\sum_{j} \mathcal{L}_{i j} \rho_{j}(t) .
$$

$\rho_{i}(t)$ is the occupation probability of the $i$ state of the system and $\mathcal{L}$ is the Liouvillian superoperator that contains all the information about the system $H_{0}$ and the jumping terms between the leads and the dots $\Gamma_{i \leftrightarrow l}$. As we just want to study the steady-state properties of the system, we solve the kernel of Eq. (1) to obtain the steady-state occupations $\rho^{\mathrm{SS}}=\operatorname{Ker}[\mathcal{L}]$.

By taking the steady-state occupations and the tunneling rates to and from the contacts we are able to calculate the current and the differential conductance

$$
\begin{aligned}
I & =\sum_{i, j=0}^{1} \rho_{|1, i, j\rangle}^{\mathrm{SS}} \Gamma_{|0, i, j\rangle \leftarrow|1, i, j\rangle}^{(-)}-\rho_{|0, i, j\rangle}^{\mathrm{SS}} \Gamma_{|1, i, j\rangle \leftarrow|0, i, j\rangle}^{(+)} \\
G & =\frac{d I}{d V}
\end{aligned}
$$

In order to distinguish the electrons coming from the different sources, we apply a lock-in signal which allows us to distinguish the electrons coming from the different paths. We use the label B (C) for the electrons flowing from dot B (C); hence, instead of eight states we have now 27. The current and conductance through path $\alpha$ is given by

$$
\begin{aligned}
I_{\alpha} & =\sum_{i, j=0, \mathrm{~B}, \mathrm{C}} \rho_{|\alpha, i, j\rangle}^{\mathrm{SS}} \Gamma_{|0, i, j\rangle \leftarrow|\alpha, i, j\rangle}^{(-)}-\rho_{|0, i, j\rangle}^{\mathrm{SS}} \Gamma_{|\alpha, i, j\rangle \leftarrow|0, i, j\rangle}^{(+)}, \\
G_{\alpha} & =\frac{d I_{\alpha}}{d V}
\end{aligned}
$$

where $\alpha \in\{\mathrm{B}, \mathrm{C}\}$. We have that $I=I_{\mathrm{B}}+I_{\mathrm{C}}$, so $G=G_{\mathrm{B}}+G_{\mathrm{C}}$.

\section{SIMULATION}

Using the master equation techniques described above, we calculate the transport through the two paths and the stability regions of each state. In Fig. 3(b) we plot the numerical result of the steady-state occupations $\rho_{i}^{\mathrm{SS}}$. For small and large $U_{\mathrm{G} 1}$ and $U_{\mathrm{G} 3}$ the states $|0,0,0\rangle$ and $|1,1,1\rangle$ are occupied, respectively. Above $|0,0,0\rangle$, for larger values of $U_{\mathrm{G} 3}$, the state $|0,0,1\rangle$ with one more electron in $\mathrm{C}$ becomes occupied and at the right-hand side, for larger values of $U_{\mathrm{G} 1}$, the state $|0,1,0\rangle$ with one more electron in $\mathrm{B}$ becomes occupied. Below $|1,1,1\rangle$, for smaller values of $U_{\mathrm{G} 3}$, the occupation of $|1,1,0\rangle$ with one less electron in $\mathrm{C}$ increases and at the left-hand side, for smaller values of $U_{\mathrm{G} 1}$, the state $|1,0,1\rangle$ with one less electron in B is occupied. All these regions obtained numerically correspond perfectly to the ones in Fig. 3(a). The small regions of $|1,0,0\rangle$ and $|0,1,1\rangle$ connect the states with one electron and two electrons, respectively. Each of these small regions, not really seen in Fig. 3(a), contain two TPs of path 1 and two TPs of path 2 . When two TPs coincide we have a quadruple point. 
With the information from the theoretical calculation of the occupations we determine the degenerate states present in the TPs [Fig. 3(a)] of path $1,(|0,0,0\rangle,|0,1,0\rangle,|1,0,0\rangle)$, $(|1,1,0\rangle,|1,0,0\rangle,|0,1,0\rangle)$, and $(|0,0,1\rangle,|0,1,1\rangle,|1,0,1\rangle)$, $(|1,1,1\rangle,|1,0,1\rangle,|0,1,1\rangle)$. The two last TPs are equal to the two first but with one more electron in $\mathrm{C}$, which shifts the energy of the degenerate states by Coulombic interaction. Similarly, for path 2 we have high positive differential conductance at the TPs which contain the degenerates states $(|0,0,0\rangle,|0,0,1\rangle$, $|1,0,0\rangle),(|1,0,1\rangle,|0,0,1\rangle,|1,0,0\rangle)$ and, with one more electron in $\operatorname{dot} \mathrm{B},(|0,1,0\rangle,|0,1,1\rangle,|1,1,0\rangle),(|1,1,1\rangle,|0,1,1\rangle,|1,1,0\rangle)$. There is high positive differential conductance visible in an extended area around the TPs due to strong coherence effects near the TPs and temperature broadening of the states. In path 1 the TPs thus merge and form a vertical line of high differential conductance.

In Fig. 4 we plot the measured conductance of paths 1 and 2 separately [(a),(b)] as well as the results from the simulation $[(\mathrm{c}),(\mathrm{d})]$. In path $1(2)$ we observe the splitting of the resonance between the dots $\mathrm{A}, \mathrm{B}(\mathrm{A}, \mathrm{C})$ due to the interaction with dot $\mathrm{C}(\mathrm{B})$. We also observe negative differential conductance in path 1 [gray color in Figs. 4(a) and 4(c)], where path 2 has high conductance [red color in Figs. 4(b) and 4(d)] at a TP. It is visible not only at the TPs but in an extended area around
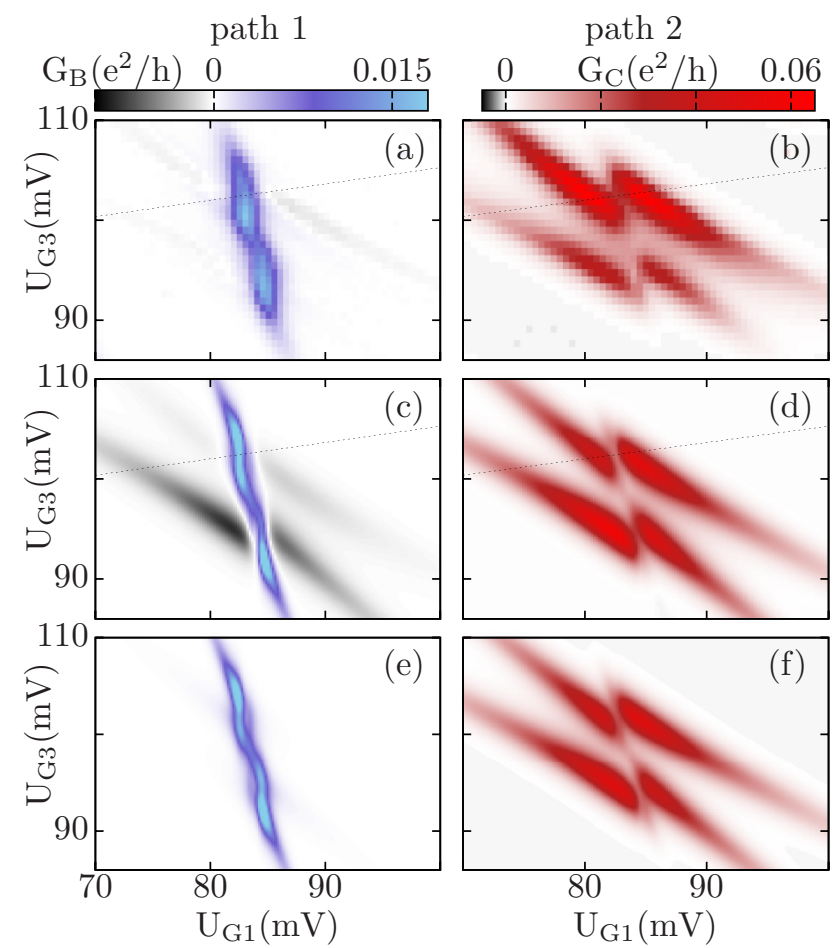

FIG. 4. (a, b) Experimental differential conductance measured at the drain contact along path 1 (a) and path 2 (b) for the setup shown in Fig. 1(a). (c, d) Theoretical simulation result reproducing the experimental figures (a) and (b), respectively. The dotted line is the cut plotted in Fig. 5. Negative differential conductance (gray regions) is visible in both simulation and experimental result. (e, f) Simulation of the differential conductance for switched transport direction measured at $S_{1}$ for path 1 and at $S_{2}$ for path 2 , respectively. Negative differential conductance is not observed because the particles are coming into the system from the same lead D. due to temperature broadening and strong coherence effects near the TPs. In the following we will analyze and compare the transport features of the two paths in more detail.

\section{CHANNEL BLOCKADE}

In Fig. 5 we show a cut from Fig. 4. We observe that the resonance of path 2 splits into two $(\boldsymbol{\Lambda}, \boldsymbol{\vee})$ due to the interaction with the third dot present in path 1 , as the energy levels of dots $\mathrm{B}$ and $\mathrm{A}$ in path 1 come into resonance at $\boldsymbol{\nabla}$. Thus, the current increases through path 1 , which partially blocks path 2 and decreases its conductance. This point is a quadruple point, i.e., four states of the two paths are coexisting in the same region of the stability diagram (see Fig. 3). The transport through path 2 is stronger than through path $1\left(\tau_{\mathrm{AC}}>\tau_{\mathrm{AB}}\right)$; thus when the quantum-dot levels in path 2 are in resonance $(\boldsymbol{\Delta}, \boldsymbol{\nabla})$ path 1 is fully blocked, decreasing its differential conductance even to negative values [more appreciable in Figs. 4(a) and 4(c)].

This channel blockade process is a consequence of Coulombic correlations between the charges flowing through the two transport channels which share dot A. This dynamical blockade depends on the ratio between the tunneling probabilities through the two paths. If the tunneling probability through path 1 (path 2) is high due to a resonance condition in this path, the transport through path 2 (path 1) with lower tunneling probability is blocked resulting from strong Coulombic interaction in the shared dot which does not allow double occupancy. In this dynamical process the electron occupation probability of dot A by electrons from one path determines the conductance through the other path. If the bias voltage is increased, the path with higher conductance raises the electron occupation of $\operatorname{dot} \mathrm{A}$, making dot A less accessible to electrons from the other path. Therefore, the transport of the other path decreases with the increase of the bias voltage, which explains the negative differential conductance observed in the stability diagrams.

In Fig. 6 we schematically identify the dominating and the blocked transport channels for each resonance $(\boldsymbol{\Lambda}, \boldsymbol{\nabla}, \boldsymbol{\nabla})$. We show the involved initial and final states connected by two different transport paths. Transport through path 1 connecting two different charge states of the system is illustrated by blue arrows; transport through path 2 is illustrated by red arrows. In the case of the resonant configuration, $(\boldsymbol{\Delta})$ and $(\boldsymbol{\nabla})$, transport

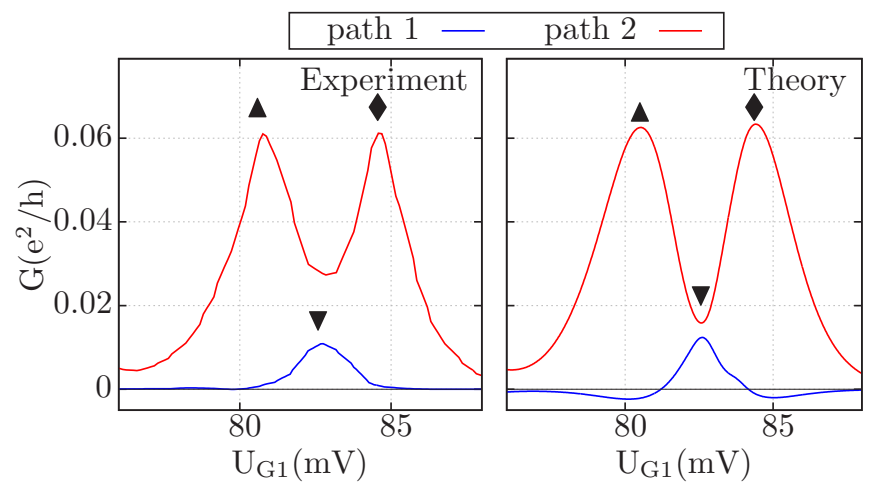

FIG. 5. Cut through the transport measurement (left) and simulation (right) of Fig. 4 for paths 1 and 2 at $U_{\mathrm{G} 3}=103 \mathrm{mV}$. 
$(\mathbf{\Lambda})$

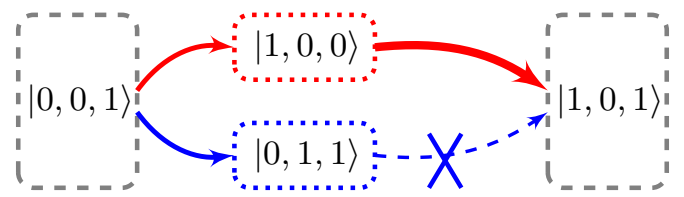

$(\mathbf{v})$

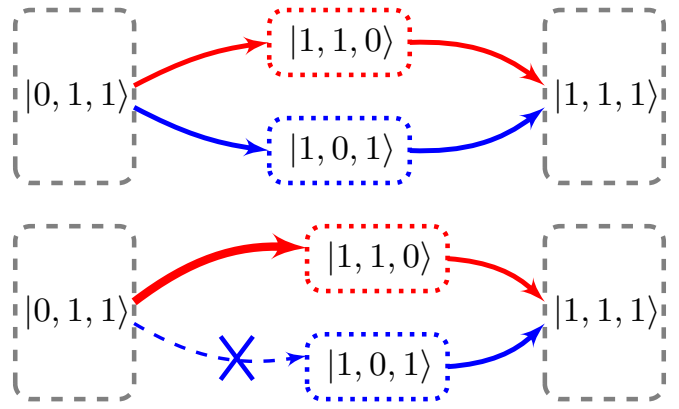

FIG. 6. Transport mechanism for the peaks in Fig. 5. In $(\boldsymbol{\Lambda}, \diamond)$ path 2 blocks path 1, schematically shown with a cross in a dashed line. In $(\boldsymbol{\nabla})$, however, dot A becomes partially occupied with electrons coming from both paths.

through path 1 is blocked by transport through path 2 . In the resonant configuration $(\boldsymbol{\nabla})$ both paths conduct and the occupation of $\operatorname{dot} \mathrm{A}$ is provided by the transport from both paths.

To observe the channel blockade in our experiment, it is crucial to have two sources $[(+)$ transport direction, indicated in Fig. 1(a)]. If we switch the transport direction of both paths with a source lead connected to dot $\mathrm{A}$ and two drain leads connected to dots B and C [(-) transport direction], the electron flow splits at dot A into two paths with a probability which depends on the tunneling rates of path $1\left(\tau_{\mathrm{AB}}, \Gamma_{\mathrm{S}_{1}}\right)$ and path $2\left(\tau_{\mathrm{AC}}, \Gamma_{\mathrm{S}_{2}}\right)$; hence there is no blocking effect between the paths. While negative differential conductance is visible in the experiment and simulation detecting the current at the common drain lead of the paths [Figs. 4(a), 4(b), and 4(c), 4(d), respectively], no negative differential conductance occurs for the switched bias direction, as can be seen in Figs. 4(e) and 4(f).

Consequently, the transport directions in the two-path device are not equivalent, as shown in Fig. 4. Thus, the transport through the dot setup is nonlinear even around zero bias voltage. The two transport directions in such a two-path device are equivalent only for an equal tunneling probability in both paths. The nonlinearity of transport in our device with unequal tunneling rates in the paths $\left(\tau_{\mathrm{AB}}<\tau_{\mathrm{AC}}\right.$ and $\left.\Gamma_{\mathrm{S}_{1}}<\Gamma_{\mathrm{S}_{2}}\right)$ is shown in Fig. 7, where we plot the differential conductance of path 1 [Fig. 7(a)] and path 2 [Fig. 7(b)] in an area around a triple-dot resonance as a function of the bias voltage at path $1, U_{\mathrm{S} 1}$, and gate voltage $U_{\mathrm{G} 3}$. A Coulomb diamond of dot $\mathrm{A}$ is visible. We observe high differential conductance in both of the paths at zero bias voltage and $U_{\mathrm{G} 3}=180.2 \mathrm{mV}$. This region corresponds to a quadruple point. Thus we have a similar situation as in Fig. 5. A cut through this region in path 1 and path 2 shows the differential conductance through the paths separately in dependence of the bias voltage at path 1 , which determines the transport direction in this path. The transport through path 1 exhibits a Coulombic peak being asymmetric around zero bias voltage (Fig. 8); the transport through path 2 shows a corresponding asymmetric dip. The asymmetry
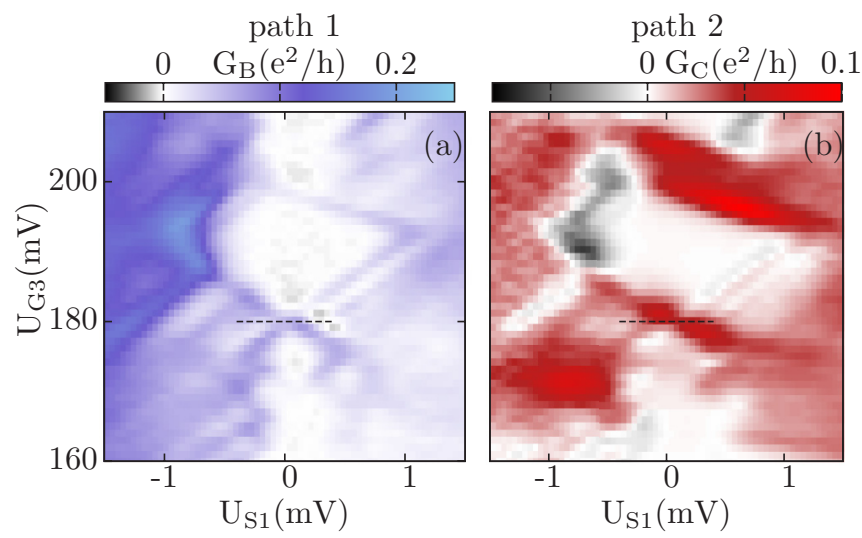

FIG. 7. Differential conductance of path 1 (a) and path 2 (b) as a function of bias voltage at $S_{1}$. We observe high differential conductance in both paths at zero bias voltage and $U_{\mathrm{G} 3}=180.2 \mathrm{mV}$, where we expect to have a quadruple point.

stems from the inequality of the two transport directions of the system originating from the direction-dependent channel blockade effect. It clearly shows that the transport is highly nonlinear, even around zero bias voltage.

The charge transport blockade was previously studied theoretically for one dot attached to three leads (one drain and two sources) which contain some amount of up and down spins [28]. Electron spins coming from different leads compete to occupy the dot, blocking the access of the other electron spin coming from the other lead. Other configurations [29-31] where channel blocking occurs consist of two independent parallel capacitively coupled double quantum dots. Here, in contrary, the states of the dots are coherently coupled forming molecular states so that the electrons are delocalized throughout the TQD system and consequently in both paths. Hence the transport through the TQD structure involves quantum coherences between the states occupied by electrons flowing through the different paths.

While former experimental work investigated basic transport properties through a triple quantum dot with only one source lead [4], here we analyze in detail a different transport configuration where two source and one drain leads
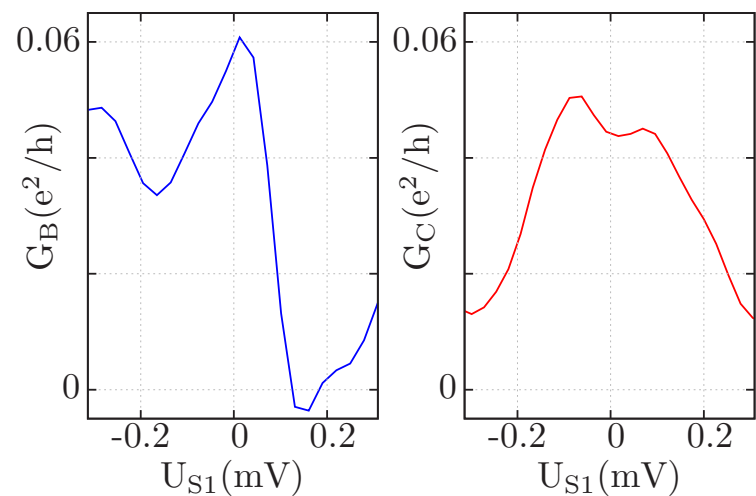

FIG. 8. Cuts (a) along the dotted line [Fig. 7(a)] in path 1 and (b) along the dotted line [Fig. 7(b)] in path 2 close to a quadruple point. The differential conductance in path 1 (path 2) shows an asymmetric peak (dip) which reflects nonlinear transport around zero bias voltage. 
combined with two different lock-in frequency signals allow us to investigate nonlinear transport characteristics through the two different paths mediated by interchannel Coulombic interactions. Only in this way does the channel blockade manifest itself in the conductance, as it is dependent on the direction of electron flow in each path. With the support of the simulation and bias measurements, we are able to show that the transport indeed is nonlinear in this system.

\section{v. CONCLUSION}

In summary, we have shown channel blockade in electronic transport through a TQD system with two source leads, which leads to nonlinear transport characteristics in such a setup. Interchannel Coulombic interaction between electrons coming from the two sources gives rise to a blockade of transport through one path, when the other path has high conductance, and affects in this way the transport properties of the multiterminal device. Our work provides a step towards a better understanding of transport properties in complex multidot systems.

\section{ACKNOWLEDGMENTS}

We are grateful to M. C. Rogge for producing the triple-quantum-dot sample. We acknowledge discussions with R.Sánchez and financial support from the Spanish Ministry of Economy and Competitiveness (Contract No. MAT201458241-P).
[1] A. Vidan, R. Westervelt, M. Stopa, M. Hanson, and A. Gossard, Appl. Phys. Lett. 85, 3602 (2004).

[2] L. Gaudreau, S. A. Studenikin, A. S. Sachrajda, P. Zawadzki, A. Kam, J. Lapointe, M. Korkusinski, and P. Hawrylak, Phys. Rev. Lett. 97, 036807 (2006).

[3] D. Schröer, A. D. Greentree, L. Gaudreau, K. Eberl, L. C. L. Hollenberg, J. P. Kotthaus, and S. Ludwig, Phys. Rev. B 76, 075306 (2007).

[4] M. C. Rogge and R. J. Haug, Phys. Rev. B 77, 193306 (2008).

[5] B. Michaelis, C. Emary, and C. Beenakker, Europhys. Lett. 73, 677 (2006).

[6] C. Pöltl, C. Emary, and T. Brandes, Phys. Rev. B 80, 115313 (2009).

[7] M. Busl, R. Sánchez, and G. Platero, Phys. Rev. B 81, 121306 (2010).

[8] C. Emary, Phys. Rev. B 76, 245319 (2007).

[9] R. Sánchez, F. Gallego-Marcos, and G. Platero, Phys. Rev. B 89, 161402(R) (2014).

[10] F. Gallego-Marcos, R. Sánchez, and G. Platero, J. Appl. Phys. 117, 112808 (2015)

[11] M. Busl, G. Granger, L. Gaudreau, R. Sánchez, A. Kam, M. Pioro-Ladrière, S. A. Studenikin, P. Zawadzki, Z. R. Wasilewski, A. S. Sachrajda, and G. Platero, Nat. Nanotechnol. 8, 261 (2013).

[12] F. R. Braakman, P. Barthelemy, C. Reichl, W. Wegscheider, and L. M. K. Vandersypen, Nat. Nanotechnol. 8, 432 (2013).

[13] R. Sánchez, G. Granger, L. Gaudreau, A. Kam, M. PioroLadrière, S. A. Studenikin, P. Zawadzki, A. S. Sachrajda, and G. Platero, Phys. Rev. Lett. 112, 176803 (2014).

[14] L. Gaudreau, G. Granger, A. Kam, G. C. Aers, S. A. Studenikin, P. Zawadzki, M. Pioro-Ladrière, Z. R. Wasilewski, and A. S. Sachrajda, Nat. Phys. 8, 54 (2012).
[15] D. DiVincenzo, D. Bacon, J. Kempe, G. Burkard, and K. Whaley, Nature (London) 408, 339 (2000).

[16] M. Stopa, Phys. Rev. Lett. 88, 146802 (2002).

[17] C. Lent, P. Tougaw, W. Porod, and G. Bernstein, Nanotechnology 4, 49 (1993).

[18] M. C. Rogge and R. J. Haug, New J. Phys. 11, 113037 (2009).

[19] A. D. Greentree, J. H. Cole, A. R. Hamilton, and L. C. L. Hollenberg, Phys. Rev. B 70, 235317 (2004).

[20] H. Sasakura, S. Adachi, S. Muto, T. Usuki, and M. Takatsu, Semicond. Sci. Technol. 19, S409 (2004).

[21] M. Ishii and K. Matsumoto, Jpn. J. Appl. Phys. 34, 1329 (1995).

[22] R. Held, T. Vancura, T. Heinzel, K. Ensslin, H. Holland, and W. Wegschneider, Appl. Phys. Lett. 73, 262 (1998).

[23] U. Keyser, H. Schumacher, U. Zeitler, R. Haug, and K. Eberl, Appl. Phys. Lett. 76, 457 (2000).

[24] J. M. Elzerman, R. Hanson, J. S. Greidanus, L. H. Willems van Beveren, S. De Franceschi, L. M. K. Vandersypen, S. Tarucha, and L. P. Kouwenhoven, Phys. Rev. B 67, 161308(R) (2003).

[25] P. W. Anderson, Phys. Rev. 124, 41 (1961).

[26] H. P. Breuer and F. Petruccione, The Theory of Open Quantum Systems (Oxford University Press, Oxford, 2002).

[27] T. H. Stoof and Y. V. Nazarov, Phys. Rev. B 53, 1050 (1996).

[28] A. Cottet, W. Belzig, and C. Bruder, Phys. Rev. B 70, 115315 (2004).

[29] R. Hussein and S. Kohler, Phys. Rev. B 89, 205424 (2014).

[30] C. Nietner, G. Schaller, C. Pöltl, and T. Brandes, Phys. Rev. B 85, 245431 (2012).

[31] G. Schaller, G. Kießlich, and T. Brandes, Phys. Rev. B 82, 041303 (2010). 